\title{
MRI reveals altered brain activity and anatomy in men with CP/CPPS
}

Chronic prostatitis/chronic pelvic pain syndrome (CP/CPPS) is a heterogeneous pain condition that affects up to $10 \%$ of men. The etiology and disease mechanisms are poorly understood, and seem to differ markedly between patients. Features of the disease include local inflammation, endocrine involvement and voiding dysfunction. Whether specific changes occur in brain anatomy or function is not known, and has not been investigated in detail. Data from studies of other chronic pain conditions indicate that specific brain abnormalities are present, suggesting that the processing of pain is altered by persistent nociception. For their study published in the Journal of Urology,

Melissa Farmer and colleagues used anatomical and functional MRI (fMRI) to examine changes in brain structure and function in response to spontaneous pelvic pain in CP/CPPS patients.

The study included 19 right-handed men with CP/CPPS (mean age 36.9 years) and NIH-CPSI (NIH chronic prostatitis symptom index) score $\geq 15$, and 16 sex-matched and age-matched healthy controls. Patients underwent fMRI during spontaneous pain while inside the scanner, and continually rated fluctuations in their pain on a scale of 0 to 10 using a finger-span logging device. A visual control task was also performed, which required the same cognitive demands (attention, motor control and evaluations of magnitudes) but without the presence of CP/CPPS pain. This allowed

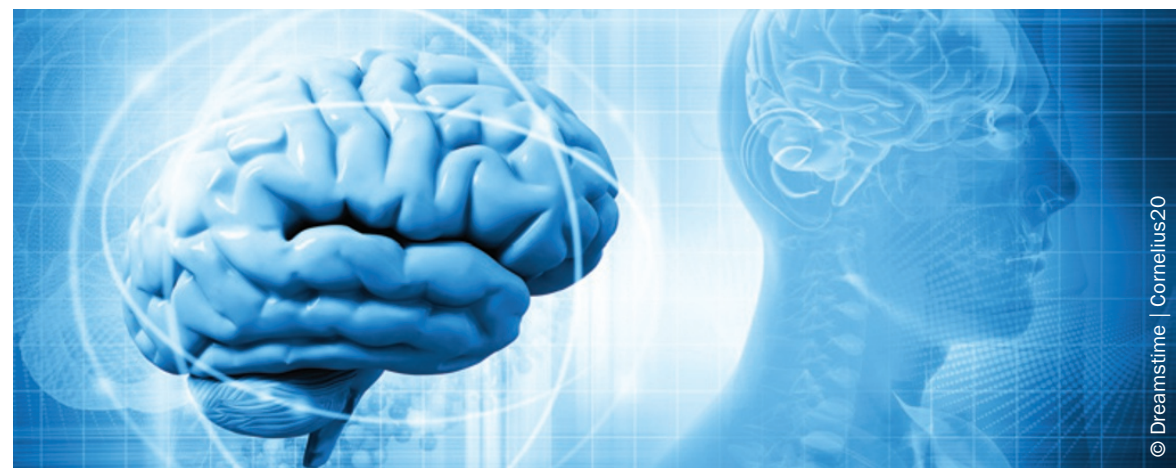

comparison of the areas of brain activity between the two tasks, and facilitated identification of the areas more-specifically activated during pain. T1-weighted and diffusion tensor imaging (DTI) were also performed to compare gray matter and white matter characteristics, respectively.

fMRI brain studies indicated activation in the right anterior insula and the secondary somatosensory cortex during pain. Anterior insula activity showed a significant positive correlation with self-reported CP/CPPS pain intensity $(r=0.57, P<0.05)$ and MPQ (McGill pain questionnaire) scores ( $r=0.78$, $P<0.01)$. Gray matter density in the anterior insula correlated positively with pain intensity $(r=0.69, P<0.01)$, and gray matter volume in the anterior cingulate cortex correlated positively with chronic pelvic pain duration $(r=0.69, P<0.01)$. Finally, the strong positive correlation between whole-brain white matter anisotropy and whole-brain gray matter volume seen in control individuals ( $r=0.87, P<0.01)$ was not present in men with CP/CPPS $(r=0.01$, $P=0.97)$, indicating a global structural disorganization between white and gray matter in these patients.

The authors provide the first evidence of altered brain activity and anatomy specific to CP/CPPS-related pain. These findings suggest that brain circuitry undergoes reorganization in a manner similar to that in other chronic pain syndromes; however, whether the observed changes are a result of the chronic presence of pelvic pain or represent predisposing factors for the development of CP/CPPS is unclear. Further studies with larger populations are required to account for the wide clinical variation in patients with $\mathrm{CP} / \mathrm{CPPS}$.

\section{Nick Warde}

Original article Farmer, M. A. et al. Brain functional and anatomical changes in chronic prostatitis/chronic pelvic pain syndrome. J. Urol. 186, 117-124 (2011) 